\title{
Estudio comparativo de la calidad biofarmacéutica de Alprazolam 0,5 mg comercializadas en el mercado peruano
}

\author{
Lennin Roswell Rodriguez-Saavedra ${ }^{1}$, Pedro Marcelo Alva-Plasencia ${ }^{2}$, Junior \\ Antonio Malca-Chamache ${ }^{2}$, Ericka Milagros Ricardo-Floriano ${ }^{2}$, José Fernando \\ Caballero-Arana ${ }^{3}$ \\ ${ }^{1}$ Universidad Nacional Mayor de San Marcos, Facultad de Medicina. Lima, Perú \\ ${ }^{2}$ Universidad Nacional de Trujillo, Facultad de Farmacia y Bioquímica. Trujillo, Perú \\ ${ }^{3}$ Universidad de San Pedro, Chimbote, Facultad de Medicina Humana. Chimbote, Perú
}

Cómo referenciar este artículo/ How to reference this article:

\begin{abstract}
Rodriguez-Saavedra LR, Alva-Plasencia $P M$, Malca-Chamache JA, Ricardo-Floriano EM, Caballero-Arana JF. Estudio comparativo de la calidad biofarmacéutica de Alprazolam $0,5 \mathrm{mg}$ comercializadas en el mercado peruano.Mem. Inst. Investig. Cienc. Salud. 2020; 18(3): 43-54
\end{abstract}

\section{RES U M E N}

El Alprazolam pertenece a las benzodiazepinas. Sus efectos se atribuyen a que actúa sobre receptores de membrana específicos, lo cual facilita la acción inhibitoria presináptica y postsináptica del ácido $y$-aminobutírico (GABA), especialmente en la formación reticular ascendente. Se utiliza para el tratamiento de los estados de ansiedad, crisis de angustia, ataques de pánico y estrés intenso. Este estudio se realizó para analizar los parámetros comparativos de control de calidad in vitro mediante la evaluación de la variación de peso, friabilidad, dureza, tiempo de desintegración, perfil y eficiencia de disolución entre el medicamento innovador (Xanax®) y multifuentes que son comercializados en el mercado peruano. Para realizarlo, se seleccionaron tabletas de Alprazolam $0,5 \mathrm{mg}$ multifuente de diferentes laboratorios comparándolos con el medicamento innovador y se evaluaron las características fisicoquímicas y biofarmacéuticas. Los ensayos farmacopeicos se evaluaron según lo establecido en la USP 42. Los resultados de las pruebas fisicoquímicas indicaron que las muestras analizadas no tenían diferencia significativa y estaban dentro de lo establecido en la farmacopea, así mismo el perfil y eficiencia de disolución permitieron establecer que el comportamiento biofarmacéutico de las mismas era muy similar para ambos tipos de molécula. Se estableció que las tabletas multifuentes de Alprazolam 0,5 mg de esta investigación son bioequivalentes con el innovador, por lo que permite proponer a la comunidad científica la determinación de la equivalencia biofarmacéutica como elemento de apoyo en la toma de decisiones de compra en el servicio farmacéutico.

Palabras clave: Alprazolam, intercambiabilidad, bioequivalencia, control de calidad.

\section{Comparative study of the biopharmaceutical quality of Alprazolam $0.5 \mathrm{mg}$ commercialized in the Peruvian market}

\begin{abstract}
A B S T R A C T
Alprazolam belongs to benzodiazepines. Its effects are attributed to the fact that it acts on specific membrane receptors, which facilitates the presynaptic and postsynaptic inhibitory action of $\mathrm{y}$-aminobutyric acid (GABA), especially in the
\end{abstract}

Financiación: Sin financiamiento.

Fecha de recepción: Junio 2020. Fecha de aceptación: Octubre 2020

*Autor correspondiente: Lennin Roswell Rodriguez-Saavedra. Facultad de Medicina, Universidad Nacional Mayor de San Marcos, Av. Grau 755, Lima, Perú.

Email: lennin.rodriguez@unmsm.edu.pe 
ascending reticular formation. It is used to treat anxiety states, panic attacks, and intense stress. This study was carried out to analyze comparative parameters of in vitro quality control by evaluating the variation in weight, friability, hardness, disintegration time, profile and dissolution efficiency between the innovative drug (Xanax $\AA$ ) and multi-sources tablets that are marketed in the Peruvian market. To perform this, Alprazolam $0.5 \mathrm{mg}$ multi-source tablets were selected from different laboratories comparing them with the innovative medicine and the physicochemical and biopharmaceutical characteristics were evaluated. Pharmacopoeial trials were evaluated as established in USP 42. The results of physicochemical tests indicated that analyzed samples did not have a significant difference and were within the established in the pharmacopoeia, as well as the profile and dissolution efficiency allowed to establish that their biopharmaceutical behavior was very similar for both types of molecules. It was established that Alprazolam $0.5 \mathrm{mg}$ multi-source tablets from this research are bioequivalent with innovator, which makes it possible to propose to scientific community determination of biopharmaceutical equivalency as a support element in decision-making process for purchasing services pharmacist.

Keywords: Alprazolam, interchangeability, bioequivalence, quality control.

\section{INTRODUCCIÓN}

Los medicamentos son esenciales para la curación, atenuación, tratamiento, prevención o diagnóstico de las enfermedades o sus síntomas. A diferencia de un bien de consumo, su calidad no es evidente para el profesional prescriptor y el paciente, por lo que es la autoridad sanitaria quien debe dar garantía de que los medicamentos que autoriza para la comercialización cumplen con los requisitos necesarios de calidad, seguridad y eficacia. Los medicamentos innovadores, desarrollados por la industria farmacéutica de investigación o también llamada de innovación, se autorizan para su uso clínico bajo el respaldo de estudios amplios y rigurosos en donde se demuestra que el medicamento cumpla con los requisitos de calidad, seguridad y eficacia. Como estos estudios representan un alto costo y varios años de investigación, estos productos farmacéuticos quedan protegidos por patentes durante un número de años que permite al laboratorio recuperar la inversión y generar ganancias ${ }^{(1,2)}$.

Una vez finalizado el periodo de protección patentaria, la industria farmacéutica puede desarrollar medicamentos con el mismo principio activo, que tienen por finalidad entregar a la población una alternativa al producto innovador y que habitualmente es de costo menor para el paciente. Para que esta alternativa sea válida, este nuevo producto debe demostrar que su comportamiento es similar al innovador (lo que se conoce como producto bioequivalente) y, si logra demostrarlo, entonces los profesionales que prescriben los medicamentos tendrán la posibilidad de intercambiar estos productos con la confianza que la calidad, seguridad y eficacia de este producto nuevo será semejante a la del innovador porque serían Equivalentes Terapéuticos. Para ello, el nuevo producto deberá demostrar que ha sido fabricado de acuerdo a un sistema que asegura el control de la variabilidad en la producción de medicamentos (las Buenas Prácticas de Manufactura, BPM), que cumple con los mismos estándares de control de calidad que el producto innovador ${ }^{(1,3)}$.

El nombre químico del Alprazolam es 8-cloro-1-metil-6-fenil-4 $\mathrm{H}-(1,2,4)$ triazolo $(4,3-a)(1,4)$ benzodiacepina (Figura 1). Es un agente de triazolobenzodiazepina con actividades ansiolíticas, sedantes-hipnóticas y anticonvulsivas. El Alprazolam se une a un sitio específico distinto del neurotransmisor inhibidor del sitio de unión del ácido gamma-aminobutírico (GABA) en el complejo receptor de ionóforos de benzodiazepina - GABA- A- cloruro ubicado en las regiones límbicas, talámicas e hipotalámicas del sistema nervioso central (SNC). Esta unión provoca una modificación alostérica del receptor y aumenta la afinidad de GABA por el receptor, lo que conduce a un aumento en la frecuencia de los eventos de apertura del canal de cloruro. Esto conduce a un aumento en el ion cloruro y produce conductancia, hiperpolarización neuronal, inhibición del potencial de acción y conduce a una disminución de la excitabilidad neuronal $^{(4-6)}$ (Figura 1). 


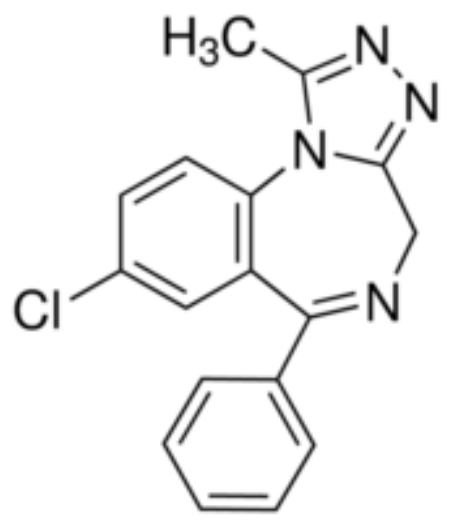

Figura 1. Fórmula estructural de Alprazolam, USP 42.

La seguridad, efectividad y eficacia de la forma de dosificación farmacéutica se puede garantizar cuando su calidad es confiable y para confirmar la calidad de la forma de dosificación farmacéutica se requiere realizar las pruebas de evaluación según los libros oficiales como la farmacopea Americana (USP) ${ }^{(7,8)}$.

Para las tabletas convencionales, la variación de peso, dureza, friabilidad, desintegración, velocidad de disolución, uniformidad de los contenidos y perfil de disolución son las pruebas de evaluación que deben realizarse para confirmar la calidad de la tableta ${ }^{(8,9)}$.

La friabilidad se prueba en una tableta para ver si la misma es estable a la abrasión o no, se prueba usando el friabilizador y se permite una pérdida máxima de peso del $1 \%$ después de la prueba de friabilidad. La prueba de variación de peso se realiza para verificar que las tabletas fabricadas tengan un peso uniforme, según lo establecido en la farmacopea americana.

La prueba de desintegración se realiza para ver cuánto tiempo tarda una tableta en descomponerse en partículas pequeñas, ya que este es el primer paso antes de la disolución del medicamento en el cuerpo. La condición de esta prueba debe ser la misma que en el cuerpo, ya que es parte de la correlación in vivo-in-vitro.

El estudio de cuantificación y uniformidad de contenido proporciona la información sobre la cantidad prácticamente disponible en la forma de dosificación dada y después de comparar con el valor teórico, se puede dar un resultado sobre la eficacia.

El perfil de disolución proporciona la información sobre el patrón de liberación del fármaco y está muy cerca de la biodisponibilidad, por lo que proporciona información sobre la seguridad y la eficacia de la forma de dosificación. Los resultados fueron sometidos a análisis estadísticos y modelos de enfoques independientes de factor de diferencia (f1), factor de similitud (f2) y eficiencia de disolución (\% ED) también fueron empleados ${ }^{(8,10)}$. Por lo tanto, el objetivo de este trabajo fue evaluar la calidad biofarmacéutica de las tabletas de Alprazolam innovador y multifuentes comercializadas en el mercado peruano comparando los resultados de calidad.

\section{MATERIALES Y MÉTODOS}

\section{Materiales}

Todos los productos químicos utilizados fueron comprados de Merck Company. El estándar de Alprazolam fue adquirido de E. Merck, Darmstadt, Alemania. Las pruebas de cromatografía de líquidos de alto rendimiento (HPLC) se realizaron en un HPLC-20 AT pump, SPD-20 A UV/visible detector (Shimadzu, Japón) empleando membranas Millipore $(0,45)$ fabricadas en EEUU. Se utilizaron equipos para medir la friabilidad, la dureza, el tiempo de desintegración y la disolución como: el medidor de friabilidad (Veegofriabilator VFT-2, USA), los aparatos de disolución (TDT-08L, Electrolab, USA), el probador de dureza (8M, Dr. Schleuniger, Switzerland), el aparato de desintegración ((Model: VDT-2, Veego USA). Una balanza analítica (Model: AY-200, SHIMADZU Corporation, Japan) se utilizó para medir la variación de los pesos de las tabletas. Las tabletas de Alprazolam 0,5 mg (de fabricación nacional e importado) se 
Rodriguez-Saavedra $L R$, et al Estudio comparativo de la calidad biofarmacéutica de Alprazolam 0,5 mg comercializadas en el mercado peruano.

compraron en las diferentes boticas autorizadas del mercado local y se identificaron de la siguiente manera:

Tabla 1. Identificación de las muestras analizadas

\begin{tabular}{cccc}
\hline $\begin{array}{c}\text { CODIGO } \\
\text { INTERNO }\end{array}$ & N $^{\circ}$ LOTE & FECHA DE FABRICACIÓN & $\begin{array}{c}\text { FECHA DE } \\
\text { VENCIMIENTO }\end{array}$ \\
\hline APZR & 10668609 & $03-2019$ & $04-2022$ \\
APZM1 & 20290490 & $01-2019$ & $02-2022$ \\
APZM2 & 20164680 & $12-2018$ & $02-2023$ \\
\hline
\end{tabular}

\section{Medición de la dureza de las tabletas de Alprazolam (prueba de dureza)}

Sobre la base del método publicado en la USP 42, se tomaron por separado 20 tabletas de cada uno de los tipos utilizados en esta investigación de Alprazolam innovador (APZR), Alprazolam multifuente 1 (APZM1) y Alprazolam multifuente 2 (APZM2) (Tabla 2).

El grado de dureza de cada tipo se midió mediante el siguiente procedimiento: Para poner en funcionamiento el equipo, se seleccionó la unidad de trabajo para la dureza "(kp)" de kilopondios. Al mismo tiempo, la barra medidora se abrió para dar campo a la tableta a ensayar, retirando el protector plástico y colocando la primera tableta entre la barra estacionaria y la barra medidora (movible). La barra medidora movible inició su movimiento horizontal hacia la barra estacionaria de modo que presionó la tableta hasta provocar su ruptura. En ese momento en la pantalla se visualizó el valor de la dureza obtenida de cada tableta. La barra medidora se abrió automáticamente, dando el espacio necesario para proceder a limpiar la zona, el protector plástico y con la ayuda de una brocha se tuvo que retirar la tableta rota. Una vez realizada la limpieza, se colocó la siguiente tableta a ensayar procediendo como se indica desde el segundo punto ${ }^{(11)}$.

\section{Medición de la friabilidad de las tabletas de Alprazolam (prueba de friabilidad)}

Sobre la base de los métodos informados en la USP 42, para tabletas con un peso unitario igual o menor a $650 \mathrm{mg}$, se tomó una muestra de tabletas enteras correspondiente lo más cercano posible a $6,5 \mathrm{~g}$. Para tabletas con un peso unitario mayor a $650 \mathrm{mg}$, se tomó una muestra de 10 tabletas enteras. Debió quitarse el polvo de las tabletas cuidadosamente antes de realizar la prueba. Se pesó con exactitud la muestra de tabletas y se colocó en el tambor, éste se hizo girar a $25 \mathrm{rpm} \times 4$ minutos y se retiraron las tabletas. Se quitó el polvo suelto de las tabletas como se hizo anteriormente y se pesó con exactitud. Cada conjunto de tabletas se colocó simultáneamente en el instrumento Friability Tester. Se calcularon los porcentajes de friabilidad de los comprimidos. Generalmente la prueba se realiza una vez. Si se encuentran tabletas claramente agrietadas, segmentadas o rotas en la muestra de tabletas después de la prueba, la muestra no ha pasado la prueba. Para este caso se consideró aceptable una pérdida media máxima de peso de la muestra utilizada de no más de $1,0 \%{ }^{(11)}$.

\section{Medición del tiempo de desintegración de las tabletas de Alprazolam}

El instrumento estuvo equipado con una canasta que contuvo 6 tubos de extremo abierto con una longitud de $7,5-8 \mathrm{~cm}$ y un diámetro de $2,15 \mathrm{~cm}$. Un tamiz de acero inoxidable de malla 10 se puso debajo de los tubos. En un vaso beaker de $1000 \mathrm{~mL}$ de capacidad se colocó el medio de desintegración. El volumen del líquido en el recipiente es tal que, en el punto más alto del recorrido ascendente, la malla de alambre permanece al menos $15 \mathrm{~mm}$ por debajo de la superficie del líquido y desciende a no menos de $25 \mathrm{~mm}$ del fondo del recipiente, en el recorrido descendente. En ningún momento puede quedar sumergida la parte superior del montaje canastilla-gradilla. Se debe verificar que el medio especificado como líquido de inmersión (agua purificada) 
esté a $37^{\circ} \mathrm{C} \pm 2{ }^{\circ} \mathrm{C}$. Se controló la temperatura del baño de agua manteniendo el termómetro sumergido durante todo el ensayo. Cada vez, se tomaron seis tabletas al azar de cada tipo de tabletas y a cada tubo de vidrio de extremo abierto, se colocó una tableta y se cubrió con un disco de plástico de 9,35-9,65 $\mathrm{mm}$ de espesor y 20,55-20,85 mm de diámetro. El disco está hecho de un material plástico transparente adecuado, con un peso específico entre 1,18-1,20. Luego, se encendió el instrumento y se registró el tiempo de desintegración de cada tableta. Se determinaron y registraron el tiempo de desintegración mínimo, máximo y promedio de cada tipo de tableta. El tiempo mínimo de desintegración corresponde al momento en que la primera tableta comenzó a desintegrarse y el tiempo máximo de desintegración fue el momento en que la última tableta comenzó a desintegrarse. De acuerdo con la regla general, las seis tabletas recubiertas en agua destilada deben desintegrarse en un período de hasta 15 minutos. Se considera que las muestras se han desintegrado y se registra el tiempo de desintegración cuando: no quedan residuos en la canastilla, los residuos de la muestra (forma farmacéutica sólida evaluada) constituyen una masa blanda sin presencia de contenido (polvo, gránulos, etc.) y cuando quedan fragmentos insolubles del recubrimiento, que permanecen en la canastilla o se adhieren a la superficie del $\operatorname{disco}^{(11)}$.

\section{Variación de peso}

Se seleccionaron al azar 20 tabletas de cada uno de los tipos de tabletas y se pesaron con una balanza analítica precisa. De acuerdo con las referencias auténticas, el rango aceptable debe estar dentro del $92,5-107,5 \%$ del peso del medio ${ }^{(11)}$.

\section{Preparación de solución amortiguadora y solución madre}

Para preparar la solución amortiguadora se disolvieron $80 \mathrm{~g}$ de fosfato monobásico de potasio y $20 \mathrm{~g}$ de fosfato dibásico de potasio en $1 \mathrm{~L}$ de agua destilada. Se agregó, mezclando, solución de ácido fosfórico o de hidróxido de potasio $(0,45 \%)$, según fue necesario para ajustar la solución de modo tal, que la solución resultante tenga un $\mathrm{pH}$ de $6,0 \pm 0,1$. Para preparar la solución madre se realizó una dilución de $0,05 \mathrm{mg} / \mathrm{mL}$ de ER Alprazolam (estándar de referencia) USP en metanol. Se agregaron $50 \mathrm{~mL}$ de solución madre amortiguadora y $250 \mathrm{~mL}$ de agua a un matraz de $500 \mathrm{~mL}$. Se agregaron al matraz $5,0 \mathrm{~mL}$ de la solución anteriormente preparada por cada $0,25 \mathrm{mg}$ de Alprazolam contenido en la tableta en análisis. Finalmente, se diluyó con agua a volumen de enrase ${ }^{(11)}$.

\section{Preparación de las soluciones estándar}

Para trazar la curva de calibración, se necesitaron concentraciones de 0,2;0,4; 0,$6 ; 0,8$ y $1,0 \mathrm{mg} / \mathrm{mL}$. De la solución madre mencionada anteriormente, se tomaron $2,4,6,8$ y $10 \mathrm{~mL}$ y cada uno se colocó en un matraz volumétrico individual de $10 \mathrm{~mL}$, luego se llevaron a volúmenes exactos de $10 \mathrm{~mL}$ agregando la solución de Acetonitrilo: Cloroformo: Alcohol butílico: Ácido acético glacial: Agua (850:80:50:0.5:20V/V). Homogeneizar a cada uno de los matraces. Por lo tanto, se obtuvieron soluciones con concentraciones de 0,$2 ; 0,4 ; 0,6 ; 0,8$ y $1,0 \mathrm{mg} / \mathrm{mL}$ que se usaron para trazar la curva de calibración e inyección en el instrumento de HPLC ${ }^{(11)}$.

\section{Elaboración de la curva de calibración del estándar}

Para elaborar la curva estándar, se inyectaron 5 veces y cada vez $50 \mu \mathrm{L}$ de cada una de las soluciones estándar preparadas en el equipo de HPLC desde las concentraciones más bajas a las más altas. Los cromatogramas y los datos relevantes, como el área del pico, la altura del pico, el tiempo de retención, etc., se registraron y guardaron como tablas de informe de pico en el programa del software. Para garantizar la precisión del método de medición de todos los procedimientos para elaborar la curva de calibración se repitieron tres veces. Luego, se trazó la curva de calibración. Sobre la base de la curva de calibración, las muestras desconocidas se inyectaron en el instrumento de HPLC y los cromatogramas se registraron, luego se determinaron las cantidades de activo de las muestras desconocidas ${ }^{(11)}$. 


\section{Cuantificación del principio activo}

Se tomaron no menos de 20 tabletas y molieron a polvo fino. Se pesó con exactitud alrededor de $3200 \mathrm{mg}$ de muestra (equivalente a $10 \mathrm{mg}$ de Alprazolam), luego se transfirió a un matraz volumétrico adecuado y se agregó una cantidad de agua equivalente al $1 \%$ del volumen del matraz. Se transfirió una cantidad de solución amortiguadora preparada anteriormente equivalente al $10 \%$ del volumen del matraz, y se agitó vigorosamente durante 10 minutos y luego se diluyó con acetonitrilo a volumen. Se homogeneizó y filtró por membrana HVLP de 0,45 $\mu \mathrm{m}$ y se inyectó. Las Tabletas de Alprazolam 0,5 mg deben contener no menos de $90,0 \%$ y no más de $110,0 \%( \pm 0,05 \mathrm{mg})$ de la cantidad declarada ${ }^{(11)}$.

\section{Uniformidad del contenido}

Se utilizaron 10 comprimidos de cada tipo. Para este ensayo se agregó una tableta (equivalente a $0,5 \mathrm{mg}$ de Alprazolam) a un matraz volumétrico de $25 \mathrm{~mL}$. Se agregaron $0,4 \mathrm{~mL}$ de agua destilada directamente sobre la tableta, se dejó la tableta en reposo durante 2 minutos y luego se agitó por rotación suave el recipiente para dispersar la tableta. Se llevó a volumen con solución amortiguadora y se homogeneizó y filtró por membrana de 0,45 $\mu \mathrm{m}$ y se inyectó( ${ }^{(11)}$.

\section{Medición de la velocidad y perfil de disolución}

De acuerdo con las condiciones informadas en la monografía de Alprazolam en la farmacopea Americana USP 42, se utilizó $500 \mathrm{~mL}$ de solución amortiguadora pH 6,0 como medio de disolución a $37^{\circ} \mathrm{C} \pm 0,5^{\circ} \mathrm{C}$, aparato tipo 1 (cesta) a $100 \mathrm{rpm}$. Según las especificaciones de la farmacopea mencionada la cantidad de fármaco disuelto deber ser no menor a $80 \%$ en 45 minutos. Para el perfil de disolución, en esta investigación, se colocaron individualmente 6 tabletas de cada tipo en las cestas del equipo de disolución, se midió y calculó la extensión de la velocidad de disolución como porcentaje del ingrediente activo liberado en diferente tiempos de recolecta de muestra. Los cuales fueron $5,10,15,20,30,45$ y 60 minutos.

Fueron tomadas alícuotas utilizando una jeringa $y$, posteriormente filtradas con filtro PVDF (PVDF $25 \mathrm{~mm}$ de diámetro y $0.45 \mu \mathrm{m}$ de tamaño de poro). La cuantificación del fármaco fue realizada a través de método cromatográfico de HPLC. Las condiciones utilizados para el análisis fueron: fase móvil Acetonitrilo: Tetrahidrofurano: Solución Amortiguadora (35:5:60)L7, $20 \mu \mathrm{L}$ de volumen de inyección, flujo de $1 \mathrm{~mL} / \mathrm{min}$, columna $4,6 \mathrm{~mm} \times 10 \mathrm{~cm} \mathrm{L7}$, y detector a $254 \mathrm{~nm}^{(11)}$.

\section{Eficiencia de disolución (ED\%)}

La eficiencia de disolución (ED) se empleó para comparar la liberación del fármaco de las diferentes marcas. La eficiencia de disolución es el área bajo la curva de disolución dentro de un rango de tiempo expresado como un porcentaje de la curva de disolución al máximo durante el mismo período de tiempo. Se puede decir que el medicamento innovador y el producto miltifuente son equivalentes si la diferencia entre sus eficiencias de disolución está dentro de los límites apropiados ( $\pm 10 \%$, que a menudo se usa) ${ }^{(12)}$. 
Rodriguez-Saavedra $L R$, et al Estudio comparativo de la calidad biofarmacéutica de Alprazolam 0,5 mg comercializadas en el mercado peruano.

\section{RESULTADOS}

Tabla 2. Dureza promedio (KP) y Velocidad de desintegración (min) de los comprimidos innovador y multifuente de Alprazolam 0,5 mg.

\begin{tabular}{ccccc}
\hline ALPRAZOLAM & $\begin{array}{c}\text { PROMEDIO } \\
\text { (KP) }\end{array}$ & D.S. & $\begin{array}{c}\text { PROMEDIO } \\
\text { (MIN) }\end{array}$ & D.S. \\
\hline APZR & 5,4 & 0,28 & 5,5 & 0,08 \\
APZM1 & 5,5 & 0,25 & 5,5 & 1,06 \\
APZM2 & 5,5 & 0,25 & 5,7 & 0,06 \\
\hline
\end{tabular}

Tabla 3. Peso promedio de los comprimidos innovador y multifuente de Alprazolam $0,5 \mathrm{mg}$.

\begin{tabular}{ccccc}
\hline & $\begin{array}{c}\text { PROMEDIO } \\
(\mathbf{m g})\end{array}$ & C.V. & D.S. & VARIANZA \\
\hline ALPRAZOLAM & 145,1 & $0,74 \%$ & 1,07 & 1,15 \\
APZR & 144,6 & $0,65 \%$ & 0,93 & 0,87 \\
APZM1 & 144,9 & $0,74 \%$ & 1,08 & 1,16 \\
\hline APZM2 & &
\end{tabular}

Tabla 4. Especificación establecida del porcentaje peso perdido después del ensayo de friabilidad según USP42 para los comprimidos innovador y multifuente de Alprazolam $0,5 \mathrm{mg}$.

\begin{tabular}{cccccc}
\hline ALPRAZOLAM & $\begin{array}{c}\text { PESO } \\
\text { INICIAL } \\
\text { (mg) }\end{array}$ & $\begin{array}{c}\text { PESO } \\
\text { FINAL } \\
\text { (mg) }\end{array}$ & $\begin{array}{c}\text { \% DE } \\
\text { PERDIDA }\end{array}$ & $\begin{array}{c}\text { ESPECIFICACIÓN } \\
\text { USP 42 }\end{array}$ & CUMPLE \\
\hline APZR & 6640,4 & 6631,3 & 0,14 & $\%$ DE PERDIDA & $<$ \\
APZM1 & 6721,4 & 6706,2 & 0,23 & SI \\
APZM2 & 6688,1 & 6662,8 & 0,38 & $\mathbf{1 \%}$ & SI \\
\hline
\end{tabular}

Tabla 5. Porcentaje promedio del contenido de principio activo según lo declarado (\%) y Porcentaje promedio de la cantidad disuelta de 6 tabletas para establecer la velocidad de disolución ( $\mathrm{Q} \%$ ) de los comprimidos innovador y multifuente de Alprazolam 0,5 mg.

\begin{tabular}{ccccc}
\hline ALPRAZOLAM & $\begin{array}{c}\text { PROMEDIO } \\
(\mathbf{\%})\end{array}$ & D.S. & $\begin{array}{c}\text { Q\% } \\
\text { (PROMEDIO) }\end{array}$ & D.S. \\
\hline APZR & 100,39 & 0,43 & 100 & 2,34 \\
APZM1 & 100,10 & 0,53 & 98 & 2,58 \\
APZM2 & 100,74 & 0,39 & 99 & 1,63 \\
\hline
\end{tabular}

Tabla 6. Factor de Diferencia (f1) y Factor de similitud (f2) de APZR con los APZM1 y APZM2.

\begin{tabular}{ccc}
\hline $\mathbf{f 1}$ & APZM1 & APZM2 \\
APZR & 8,07 & 4,97 \\
$\mathbf{f 2}$ & APZM1 & APZM2 \\
APZR & 54,25 & 64,16 \\
\hline
\end{tabular}


Los factores de similitud y el de diferencia se definen como unos cálculos matemáticos que permiten determinar el grado de concordancia o disparidad obtenido en los perfiles de disolución de dos formulaciones diferentes obtenidos en las mismas condiciones. Dichos factores son independientes del modelo y sus criterios de aceptación son los siguientes: Factor de diferencia ( $f 1)$ es aceptable entre 0 y 15 , siendo 0 la ideal. Lo cual indica que prácticamente no hay diferencia entre las formulaciones. Factor de similitud (f2) es aceptable entre 50 y 100 , siendo 100 el ideal. Lo cual indica que prácticamente las formulaciones son iguales, o muy parecidas.

Tabla 7. Perfil de disolución y Eficiencia de disolución (ED\%) del APZR, APZM1 y APZM2

\begin{tabular}{ccccccc}
\hline \multirow{2}{*}{ TIEMPO } & \multicolumn{2}{c}{ APZR } & \multicolumn{2}{c}{ APZM1 } & \multicolumn{2}{c}{ APZM2 } \\
\cline { 2 - 7 } & MEDIA & D.S. & MEDIA & D.S. & MEDIA & D.S. \\
\hline $\mathbf{0}$ & 0 & 0 & 0 & 0 & 0 & 0 \\
$\mathbf{5}$ & 65,64 & 4,49 & 90,04 & 1,12 & 89,91 & 4,35 \\
$\mathbf{1 0}$ & 84,38 & 0,99 & 97,51 & 0,66 & 98,86 & 1,04 \\
$\mathbf{1 5}$ & 90,61 & 1,07 & 100,04 & 1,43 & 100,61 & 0,64 \\
$\mathbf{2 0}$ & 94,68 & 0,87 & 101,33 & 1,60 & 100,73 & 0,33 \\
$\mathbf{3 0}$ & 98,07 & 1,03 & 101,40 & 3,17 & 100,29 & 0,53 \\
$\mathbf{4 5}$ & 99,74 & 0,83 & 101,72 & 2,17 & 99,59 & 0,59 \\
$\mathbf{6 0}$ & 99,29 & 0,46 & 101,94 & 2,57 & 99,45 & 0,74 \\
\hline ED\% & \multicolumn{2}{c}{89,71} & \multicolumn{2}{c}{95,09} & \multicolumn{2}{c}{93,05} \\
\hline
\end{tabular}

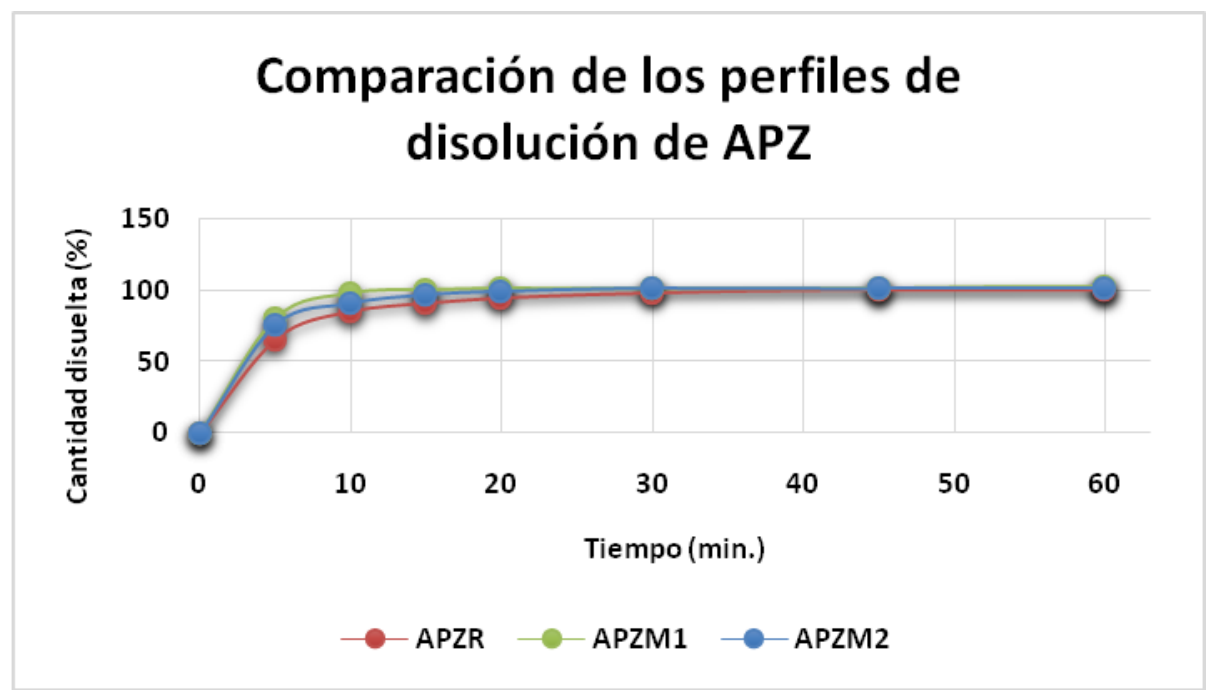

Figura 2. Perfil de disolución de tabletas de Alprazolam $0,5 \mathrm{mg}$. innovador $y$ multifuente. (APZR, APZM1 y APZM2).

\section{DISCUSIÓN}

La vía de administración oral es la ruta de administración más preferida, ya que ofrece uno de los métodos más seguros y convenientes de administración de medicamentos. Por lo tanto, es necesario evaluar el mantenimiento de la calidad perfecta de cada medicamento para la salud humana. La forma de dosificación de tabletas es una de las formas de dosificación más preferidas en todo el mundo. Casi todas las moléculas de fármacos pueden formularse en tabletas y el proceso de fabricación de tabletas es muy simple y muy flexible ${ }^{(13)}$. Además, los excipientes, tales como deslizantes, diluyentes, aglutinantes o agentes de granulación, pueden incluir lubricantes para asegurar la formación eficiente de tabletas y así como también para promover la ruptura de la tableta en el tracto digestivo; edulcorantes o sabores para 
mejorar el sabor; y pigmentos para hacer las tabletas visualmente atractivas ${ }^{(14)}$. Los requisitos previos de los productos farmacéuticos que deben ser química y farmacéuticamente equivalentes deben ser idénticos en cuanto a resistencia, calidad, pureza, perfil de liberación del ingrediente activo y también en la misma forma de dosificación, para la misma vía de administración ${ }^{(15)}$.

Todas las tabletas de Alprazolam $0,5 \mathrm{mg}$ utilizadas en esta investigación estaban dentro de su vida útil de 3 años a partir de la fecha de fabricación. No se encontraron anomalías en la apariencia física de las muestras de diferentes marcas.

Las tabletas sólidas son quizás las formas de dosificación más comúnmente usadas en la prescripción de medicamentos. La dureza de la tableta sirve tanto como un criterio para guiar el desarrollo del producto como una especificación de control de calidad. Las tabletas no deben ser demasiado duras o demasiado suaves. Una tableta extremadamente dura podría indicar un potencial de unión excesiva entre los ingredientes activos y los excipientes, lo cual podría evitar la disolución apropiada de la tableta, necesaria para una dosis exacta. Por la misma razón, una tableta más suave podría ser resultado de una unión débil y podría llevar a la desintegración prematura cuando la ingiere el paciente. Una tableta suave podría también astillarse o romperse durante las etapas de proceso en la manufactura, como el recubrimiento y el acondicionado. El límite aceptable de dureza de una tableta es de 5 a $8 \mathrm{Kp}$. Además, una fuerza entre 4 y $10 \mathrm{Kp}$ también se considera satisfactoria ${ }^{(16)}$. Se determinó la dureza de las diferentes tabletas de Alprazolam y estaba entre 5,4 y 5,5 Kp que cumple con la especificación USP (Tabla 2).

La desintegración es un proceso físico relacionado con la descomposición mecánica de una tableta en una más pequeña (partículas/gránulos), que representan la rotura de interacciones entre partículas generadas durante la compactación de la tableta en partículas granuladas del Ingrediente Farmacéutico Activo (IFA) y excipientes ${ }^{(17)}$. En términos generales, después de que el líquido moja la superficie de la tableta y penetra en los poros, la desintegración tiene lugar en dos pasos: primero, desintegración de la tableta en pequeños gránulos, y segundo, desagregación o desintegración de gránulos ${ }^{(18)}$. El primer paso es importante para la tasa de liberación inicial de drogas de la tableta. Si no ocurriera la desintegración, solo el IFA cerca de la superficie del compacto se disolvería. El aumento en el área de superficie en comparación con la tableta intacta produce una mayor velocidad de disolución ${ }^{(19)}$. Se pueden agregar desintegrantes a la formulación para promover la desintegración de la forma de dosificación cuando está en contacto con un fluido. Tales excipientes suavizan la matriz de la forma de desintegración, permitiendo que ocurra por diferentes mecanismos ${ }^{(20-23)}$. Ninguna de las muestras excedió la especificación del tiempo de desintegración que estaba entre 5,5 y 5,7 min (Tabla 2).

La uniformidad del peso de la tableta es importante porque garantiza la distribución uniforme de los ingredientes en el medicamento. La distribución desigual puede alterar la dosis en cada medicamento individual y, por lo tanto, causa muchos problemas, como la imposibilidad de alcanzar el rango terapéutico o exceder el rango terapéutico y alcanzar el rango tóxico ${ }^{(24)}$. Se observó que todas las marcas cumplen con la especificación USP que estaba entre $145,1 \mathrm{mg} \pm 0,74 \%, 144,6 \mathrm{mg} \pm 0,65 \%$ y $144,9 \mathrm{mg} \pm 0,74 \%$ (Tabla 3).

La prueba de friabilidad es un método que se emplea para determinar resistencia física de las tabletas tras exposición a choque mecánico y desgaste. En palabras simples, la prueba de friabilidad indica si las tabletas son capaces de soportar el estrés mecánico que sufre durante su fabricación, distribución y manejo por parte del paciente. La resistencia mecánica de la tableta puede determinarse por su dureza y a través de la prueba de friabilidad. La fuerza de una tableta juega un papel muy importante en su comercialización y disolución ${ }^{(25)}$. Los resultados de la prueba de friabilidad son inferiores al $1 \%$, y según la farmacopea, el límite es del $1 \%$ del peso inicial de las tabletas, por lo que todas las tabletas de Alprazolam 0,5 mg pasaron la prueba de friabilidad, lo que significa que todas estas marcas de tabletas de Alprazolam tienen buena resistencia mecánica (Tabla 4). 
La cuantificación de principio activo, se realiza con el fin de conocer si el medicamento cumple con la cantidad de IFA declarado y si está dentro de los márgenes establecidos por la farmacopea. Una elevada o poca cantidad de principio activo en el comprimido puede ocasionar una toxicidad o nula eficacia del medicamento ${ }^{(26)}$. Los resultados de las pruebas de dosaje de todas las muestras estuvieron entre 100,10 y $100,74 \%$ que cumplen con la especificación USP para la prueba de ensayo (Tabla 5).

La velocidad de disolución es un requisito para todas las formas de dosificación oral sólida y se usa durante todo el ciclo de vida de desarrollo para la liberación del producto y las pruebas de estabilidad. Es una prueba analítica fundamental utilizada para detectar cambios físicos en un ingrediente farmacéutico activo y un producto formulado. En las primeras etapas del proceso de desarrollo de fármacos, las pruebas de velocidad de disolución in vitro apuntalan la optimización de la liberación de fármacos a partir de una formulación dada ${ }^{(27)}$. Se determinó la velocidad de disolución de las diferentes tabletas de Alprazolam $0,5 \mathrm{mg}$. La especificación USP indica que debe ser mayor a $80 \%$ de la cantidad declarada en 45 minutos, los resultados mostrados indican que la disolución estuvo entre el 98 y $100 \%$ por lo que se encuentra dentro de lo establecido por la farmacopea (Tabla 5).

El perfil de disolución puede definirse como la cantidad de sustancia farmacológica que entra en la solución por unidad de tiempo en condiciones estándar de interfaz líquido/sólido, temperatura y composición del disolvente. Se puede considerar como un tipo específico de cierta reacción heterogénea en el que una transferencia de masa resulta como un efecto neto entre el escape y la deposición de moléculas de soluto en una superficie sólida. Para comparar los perfiles de disolución de las tabletas, se empleó un enfoque independiente del modelo de factor de diferencia (f1) y factor de similitud (f2). El factor de diferencia (f1) calcula el porcentaje (\%) de diferencia entre las dos curvas en cada punto de tiempo y es una medida del error relativo entre las dos curvas y el factor de similitud (f2) es una transformación de raíz cuadrada recíproca logarítmica de la suma del error al cuadrado y es una medida de la similitud en el porcentaje (\%) de disolución entre las dos curvas ${ }^{(28)}$. La Tabla 6 muestra los valores $\mathrm{f} 1, \mathrm{f} 2$ de los multfuentes con respecto a la marca innovadora elegida. En el cálculo de f2, generalmente solo se considera una medición después de que el producto comparador haya alcanzado el $85 \%$ de disolución. Los valores para f2 son más de 50 y todos los valores de 11 son menos de 15 . Por lo tanto, podemos decir que todas las marcas son equivalentes con el producto innovador.

La eficiencia de disolución (ED) también se empleó para comparar la liberación del fármaco del medicamento innovador y multifuentes. La eficiencia de disolución es el área bajo la curva de disolución dentro de un rango de tiempo ${ }^{(29)}$. La Tabla 7 muestra la eficiencia de disolución de los diferentes multifuentes junto con el producto innovador y de acuerdo a esto las tabletas de APZM1 y APZM2 son equivalentes con APZR, ya que la diferencia del \%ED (producto de innovador - producto multifuente) es inferior a $10 \%$ por lo que se consideraran como intercambiables.

Los resultados del estudio del perfil de disolución en la Tabla 7 y Figura 2 muestran que las diferentes marcas liberan aproximadamente el $100 \%$ del medicamento en una hora. Todas liberan más del $70 \%$ del principio en 5 minutos, lo que significa que el principio activo, se disuelve rápidamente en estas marcas. Los datos demuestran que el estudio de disolución de Alprazolam cumplen con los estándares de farmacopea y al no existir diferencia significativa en los diferentes tiempos de muestreo, nos permiten establecer que puede intercambiarse los medicamentos multifuentes de Alprazolam con el innovador ${ }^{(30)}$.

\section{AGRADECIMIENTOS}

A la Facultad de Medicina de la Universidad Nacional de San Marcos y a la Facultad de Farmacia y Bioquímica de la Universidad Nacional de Trujillo, especialmente a la cátedra de Tecnología Farmacéutica, Biofarmacia y Farmacocinética por el apoyo brindado con el laboratorio y los equipos. 


\section{REFERENCIAS BIBLIOGRÁFICAS}

1. Arturo S. Bioequivalencia de medicamentos: Su aplicación en Chile y garantías para el paciente. +Quimica [Internet]. 2015 [citado 15 May 2020];4(1):4-14. Disponible en: http://www.quimica.uchile.cl/wpcontent/uploads/2013/11/RevistaQu\%C3\%A DmicaVida-N\%C2\%B01.pdf

2. Brandon J, Hala M. Community pharmacists' understanding and perceptions of FDA therapeutic equivalence standards. [La comprensión y las percepciones de los farmacéuticos comunitarios sobre los estándares de equivalencia terapéutica de la FDA]. Research in Social and Administrative Pharmacy 15 2019; 77-83. Inglés.

3. Reed J, Frank S, Shelby R, James L, Meenakshi B. Comparing the Noncomparable: The Need for Equivalence Measures That Make Sense in HealthEconomic Evaluations [Comparación de lo no comparable: la necesidad de medidas de equivalencia que tengan sentido en las evaluaciones económico-sanitarias]. ValueHealth. 2019; 22(6):684-692. Inglés.

4. Edouard L, Alexandra J, Morgane R, Marie G, Pascale J, Fanny F, et al. Do older people know why they take benzodiazepines? A national French cross-sectional survey of long-term consumers. Int J Geriatr Psychiatry [Internet]. 2020. [citado $08 \mathrm{Abr}$ 2020];1-7. Disponible en: https://onlinelibrary.wiley.com/doi/epdf/10. 1002/gps.5307.

5. Tom, L, Cristín R, Carmel $M$, Justin $P$, Zaxhary $M$, Colin $B$, et al. Brief interventions targeting long-term benzodiazepineand Zdrug use in primary care: a systematic review andmeta-analysis. Addiction [Internet]. 2020. [citado 19 Abr 2020]; 2031. Disponible en: Nick M, Annemiek V, Joris V, Aurora E, JokeH, Karel A, et al. Driving performance and neurocognitive skills of long-term users of benzodiazepine anxiolytics and hypnotics. Hum Psychopharmacol Clin Exp [Internet]. 2019. [citado $19 \mathrm{Abr} 2020] ; 1-13$. Disponible en: https://onlinelibrary.wiley.com/doi/epdf/10. 1002/hup. 2715

6. Nick M, Annemiek V, Joris V, Aurora E, Joke $H$, Karel $A$, et al. Driving performance and neurocognitive skills of long-term users of benzodiazepine anxiolytics and hypnotics. Hum Psychopharmacol Clin Exp [Internet]. 2019. [citado 19 Abr 2020];1-13. Disponible en:

https://onlinelibrary.wiley.com/doi/epdf/10. 1002/hup. 2715

7. Gupta M, Saini T. Preformulation Parameters Characterization to Design, development and Formulation of Vancomycin Hydrochloride Tablets for psudomembranous Colitis. [Parámetros de preformulación: Caracterización para el diseño, desarrollo y formulación de tabletas de clorhidrato de vancomicina para la colitis pseudomembranosa]. Int J Pharma Research Dev 2009; 1: 1-7. Inglés.

8. Weifeng T, David B. Bioequivalence and Food Effect of Dapagliflozin/Saxagliptin/Metformin

Extended-release Fixed-combination Drug Products Compared With Coadministration of the Individual Components in Healthy Subjects. [Bioequivalencia y efecto alimentario de Dapagliflozina / Saxagliptina / Metformina Medicamentos de combinación fija de liberación prolongada en comparación con la administración conjunta de los componentes individuales en sujetos sanos]. Clinical Therapeutics 2019; 1545-1563. Inglés.

9. Gupta M, Pandey S, Chauhan B, Gupta N. Design, development and evaluation of rosuvastatin calcium and diltiazem hydrochloride bilayer tablet using combination concept of sustained layer with conventional layer. [Diseño, desarrollo y evaluación de tabletas de bicapa de rosuvastatina de calcio y clorhidrato de diltiazem usando el concepto de combinación de capa sostenida con capa convencional]. Turkish Journal of Pharmaceutical Sciences 2014; 11: 269-284. Inglés.

10. Dalma S, Danielle F, Tiago D, Robert A, Simon $H$. Bioequivalence of 2 Naproxen Sodium Tablet Formulations in Healthy Male and Female Volunteers. [Bioequivalencia de 2 formulaciones de tabletas de naproxeno sódico en voluntarios sanos, hombres y mujeres]. Current Therapeutic Research 90 2019; 33-38. Inglés.

11. Farmacopea de los Estados Unidos de América, Formulario Nacional, USP, USP 42NF 37, The Pharmacopeial Convention, United Book Press, Baltimore, 2019.

12. Kalakuntla U, Veerlapati M, Chepuri R. Effect of various super disintegrants on hardness, disintegration and dissolution of medicine from dosage form. [Efecto de varios superdesintegrantes sobre la dureza, desintegracion y disolucion de la medicina de la forma de dosificacion]. J Adv Sci Res. 2010; 1(1):15. Inglés.

13. Kamble $N$, Chaudhari $P$, Oswal R, Kshirsagar S, Antre RV. Innovations in tablet coating technology. [Innovaciones en tecnología de recubrimiento de tabletas]. International Journal of Applied Biology and 
Pharmaceutical Technology. 2011; 2(1):214218. Inglés.

14. Raja S, Meyer R, Campbell J. Peripheral mechanism of somatic pain. [Mecanismo periférico del dolor somático]. Anesthesiology. 1988; 68:571-590. Inglés.

15. Jakaria M, Mousa, Parvez M, Zaman $R$, Arifujjaman Sayeed $M$, Ali $M$. In vitro Comparative Study of Different Brands of Dexamethasone Tablet Available in Bangladesh. [Estudio comparativo in vitro de diferentes marcas de tabletas de dexametasona disponibles en Bangladesh]. IJPQA. 2016; 7(2):24-28. Inglés.

16. Ziye $Z$, Chenxiang $W$, Xuyong $Z$, Xuben Y, Chao Yu, Dongchuan Z, et al. Farmacocinética, bioequivalencia y estudios de seguridad de la prucaloprida en sujetos chinos sanos. American College of Clinical Pharmacology. [Internet]. 2019. [citado 15 Mar 2020]. Disponible en: https://accp1.onlinelibrary. wiley.com/doi/10 $.1002 /$ cpdd.754.

17. Markl D, Zeitler J. A review of disintegration mechanisms and measurement techniques. [Una revisión de los mecanismos de desintegración y las técnicas de medición]. Pharm. Res.; 2017, 34, 890-917. Inglés.

18. Rubinstein $\mathrm{MH}$, Bodey DM. Disaggregation of compressed tablets. [ Desglose de tabletas comprimidas]. J Pharm Sci. 2006(65): 1749-1753. Inglés.

19. Aulton M, Taylor K. Aulton's Pharmaceutics. The Design and Manufacture of Medicines. 4th ed. Churchill Livingstone. Elsevier. 2013.

20. Quodbach J, Kleinebudde P. A critical review on tablet disintegration. [Una revisión crítica sobre la desintegración de tabletas]. Pharm Dev Technol. 2016(21): 763-774. Inglés.

21. Lowenthal W. Disintegration of tablets. [ Desintegración de tabletas] ]. Pharm. Sci. 1972(61):1695-1711. Inglés.

22. LFA [internet]. U.K.: Oxford; 2020 [actualizado 05 Ene 2020; citado $04 \mathrm{Abr}$ 2020]. Disponible en: https://www.Ifatabletpresses.com/articles/o verview-of-disintegrants.

23. Desai P, Liew C, Heng S. Review of disintegrants and the disintegration phenomena. [Revisión de los desintegrantes y los fenómenos de desintegración]. J Pharm. Sci. 2016, 105: 2545-2555. Inglés. 24. Donauer $N$, Löbenberg R. A mini review of scientific and pharmacopeial requirements for the disintegration test.
[Una mini revisión de los requisitos científicos y farmacopeicos para la prueba de desintegración]. Int. J. Pharm. 2007, 345, 2-8. Inglés.

25. Frederick O, Changquan Calvin. Validation and applications of an expedited tablet friability method. [Validación y aplicaciones de un método acelerado de friabilidad de tabletas]. International Journal of Pharmaceutics 2015. 146-155. Ingles.

26. Usmangani K, Nishma M, Dimal A, Shah F, Kashyap K. Science Direct Thinlayer chromatography method for the simultaneous quantification and stability testing of alprazolam and mebeverine in their combined pharmaceutical dosage form. [Método de cromatografía en capa fina para la cuantificación simultánea y las pruebas de estabilidad de alprazolam y mebeverina en su forma de dosificación farmacéutica combinada]. Journal of Taibah University for Science 11 (2017) 66-75. Inglés.

27. Sharonia B, Steven H. Directly compressed rosuvastatin calcium tablets that offer hydrotropic and micellar solubilization for improved dissolution rate and extent of drug release. Saudi Pharmaceutical Journal [Internet]. 2019 [citado 15 Abr 2020]; 27(5):619-628. Disponible en: http://sd.searchbox.science/science/article/ pii/S1319016418303797.

28. Takafumi K, Shuichi A. Integration of In Silico Pharmacokinetic Modeling Approaches Into In Vitro Dissolution Profiles to Predict Bioavailability of a Poorly Soluble Compound. Journal of Pharmaceutical Sciences. [Internet]. 2019 [citado $15 \mathrm{Abr}$ 2020]; 108(11):3723-3728. Disponible en: http://sd.searchbox.science/science/article/ pii/S0022354919304290

29. Manali D, Prajapata Shital B, Butania Mukesh C, Gohel B. Liquisolid: A promising technique to improve dissolution efficiency and bioavailability of poorly water soluble nimodipine. [Liquisolid: una técnica prometedora para mejorar la eficiencia de disolución y la biodisponibilidad de nimodipina poco soluble en agua]. Journal of Drug Delivery Science and Technology. 2019; 53:1773-2247. Ingles.

30. Yasmine F, Hebatallah M. Eman S, Hala Z, Khadiga K. Ecofriendly Determinations of Pseudoephedrine $\mathrm{HCl}$ and Triprolidine $\mathrm{HCl}$ in Their Tablet Form: Evaluation of Dissolution Profile.[Internet].2019. [citado 10 Mar 2020]; 4(31):8946-8952. 